\title{
Atopic Dermatitis as a Potential Portal of Septic Arthritis
}

Hidenori Kyo ${ }^{1}$, Makoto Hayashi ${ }^{1}$, Yusuke Yamawaki ${ }^{1}$, Mutsumi Watanabe ${ }^{1}$, Yuki Okutani ${ }^{1}$, Masashi Kanamura ${ }^{1}$, Hisataka Takeuchi ${ }^{1}$, Satoshi Ota ${ }^{1}$, Eijiro Onishi ${ }^{1}$, Koichi Iwaki $^{1}$, Hidekazu Nishimatsu ${ }^{2}$ and Tadashi Yasuda ${ }^{{ }^{*}}$

${ }^{1}$ Department of Orthopaedic Surgery, Kobe City Medical Center General Hospital, Kobe, Japan

${ }^{2}$ Department of Orthopaedic Surgery, Tenri Hospital, Tenri, Japan

Received: May 16, 2014, Accepted: June 27, 2014, Published: July 09, 2014

*Corresponding author: Tadashi Yasuda, Department of Orthopaedic Surgery, Kobe City Medical Center General Hospital, 2-1-1 Minatojima-minaminachi, Chuo-ku, Kobe 650 0047, Japan, Tel: +81-78-302-4321; Fax: +81-78-302-7537; E-mail: tadyasu@kcho.jp

Copyright: @ $2014 \mathrm{Kyo} \mathrm{H}$, et al. This is an open-access article distributed under the terms of the Creative Commons Attribution License, which permits unrestricted use, distribution, and reproduction in any medium, provided the original author and source are credited.

\begin{abstract}
Objective: Skin lesions of atopic dermatitis are highly colonized with gram-positive bacteria, which may enter the blood stream through the broken skin barrier. This paper described the association between atopic dermatitis and septic arthritis in an attempt to emphasize the importance of a high index of clinical suspicion for the correct diagnosis of bacterial arthritis in patients with atopic dermatitis.

Methods: We reported one and two infections of sacroiliac joint and knees, respectively, in patients with atopic dermatitis. A systematic search of the literature revealed one case of septic arthritis of hip joint in association with atopic dermatitis. We discussed the clinical features and treatment of the articular infections.

Results: In combination with localized pain, elevation of white blood cells, erythrocyte sedimentation rate, and Creactive protein indicated hematogenous articular infection in the patients at the age of 13-27 with poorly controlled atopic dermatitis. Magnetic resonance imaging was useful to identify deep articular infections. Staphylococcus aureus was the predominant pathogen, while group A streptococcus was less frequently isolated. For confirmation of the causative bacteria and spectrum of antibiotic sensitivities, skin culture was helpful in case of negative identification of the pathogen from culture of synovial fluid. Treatment with antibiotics against Staphylococcus aureus and group A streptococcus with or without surgical management resulted in good outcome.
\end{abstract}

Conclusion: This case series was the first report suggesting the association between septic arthritis and atopic dermatitis. Patients with poorly controlled atopic dermatitis may be at high risk of septic arthritis because the broken skin barrier could become a potential portal of pathogens.

Keywords: Atopic dermatitis; Septic arthritis; Knee joint; Hip joint; Sacroiliac joint; Staphylococcus aureus; Streptococcus

\section{Introduction}

Acute monoarticular arthritis has multiple potential etiologies including infection, crystalloid arthropathies, rheumatoid arthritis, lupus, and trauma [1-3]. The most serious condition is septic arthritis. Septic arthritis has an annual incidence of 10 per 100,000 individuals in the United States and is more common among those with rheumatoid arthritis or a prosthetic joint, with up to 70 cases per 100,000 [4]. Septic arthritis most commonly affects the knee, which accounts for approximately $50 \%$ of cases. In decreasing order of frequency, septic arthritis also affects the hip, shoulder, and elbow [5]. Septic arthritis is usually secondary to bacteremia because the lack of basement membrane in the synovial tissue makes bacterial organisms readily enter the synovial fluid through hematogenous route. Infectious organisms can also invade the joint by direct extension from an adjacent osteomyelitis, or by direct introduction through a wound. Delayed or inadequate treatment of septic arthritis results in irreversible joint destruction with subsequent disability because articular cartilage can be destroyed within days. Permanent disability and increased mortality are associated with delayed presentations and diagnosis [6-8]. Thus, prompt diagnosis to facilitate appropriate antibiotic management of septic arthritis is critical [6].

Atopic Dermatitis (AD) is a chronic inflammatory skin disease. Its increasing prevalence is well documented and represents a major public-health problem, mostly in industrialized countries. Atopic skin lesions are highly colonized with Staphylococcus aureus. Compared with Staphylococcus aureus, group A streptococcus is less frequently infected. It is likely that patients with AD customarily scratch the skin due to severe itching, resulting in aggravation of $\mathrm{AD}$ as well as in damage to the skin barrier which induces an invasion of colonized bacteria. This process may allow a skin infection to enter the blood stream to cause bacteremia.

There has been rising awareness in the medical literature about the potential risk of endocarditis through staphylococcal bacteremia in young patients with $\mathrm{AD}$. In contrast to endocarditis, little attention has been received to septic arthritis as one possible complication in this common skin disorder. Recently, we have demonstrated acute sacroiliac joint infection in a 15-year-old boy with $\mathrm{AD}$ [9]. In addition to the previous case, we have further experienced two cases of acute bacterial infection in knee joints associated with $\mathrm{AD}$. The present paper with a literature review has described a series of septic arthritis in association with $\mathrm{AD}$ for the first time, and has attempted to 
Page 2 of 3

emphasize the importance of a high index of clinical suspicion for the correct diagnosis of such bacterial arthritis in $\mathrm{AD}$ patients.

\section{Patients and Methods}

From 2006 to 2013, one and two cases of bacterial infections in sacroiliac joint (case 1) [9] and knee joints (cases 2 and 3), respectively, were treated for $\mathrm{AD}$ patients at the Department of Orthopaedic Surgery of Kobe City Medical Center General Hospital and Tenri Hospital.

We performed a systematic search of the literature using PubMed for relevant articles between 1990 and 2013. The keywords and Medical Subject Heading (MeSH) terms used were 'atopic dermatitis', 'septic arthritis', and 'joint infection'. Screening of the reference lists revealed a case of septic arthritis of hip joint (case 4) in association with $\mathrm{AD}[10]$.

Demographic pattern, predisposing conditions, clinical presentation, co-morbidities, clinical examinations, microbiology, treatment, and outcome of the four cases of hematogenous joint infections were retrospectively reviewed. $\mathrm{AD}$ condition in the four patients was also assessed.

\section{Results}

\section{Demographic data and condition of atopic dermatitis}

The ages of patients with septic arthritis associated with $\mathrm{AD}$ ranged from 13 to 27, and the male to female ratio was 3:1. All the patients in our case series (cases 1-3) showed generalized xerosis with some excoriated plaques of erythema and crusting on the trunk and extremities. AD skin condition in the patients with hip infection (case 4) was also deteriorated. Although topical corticosteroid cream had been used for $\mathrm{AD}$ treatment in case 2, no patient of our case series received treatment by dermatologist at their first visit. Topical corticosteroid was used for AD in case 4. Other predisposing factors were not found including diabetes mellitus, malnutrition, substance abuse, human immunodeficiency virus infection, malignancy, or renal failure. All the patients had no comorbidity such as hypertension or hypertrophic cardiomyopathy (Table 1).

\begin{tabular}{|l|l|l|l|l|l|l|}
\hline Case & Sex & Age (years old) & Atopic dermatitis & Topical corticosteroid & Predisposing factor & Comorbidity \\
\hline \multicolumn{7}{|c|}{ Our case series } \\
\hline 1 & Male & 15 & poorly controlled & none & none & none \\
\hline 2 & Male & 13 & poorly controlled & used & none & none \\
\hline 3 & Male & 27 & poorly controlled & none & none & none \\
\hline & \multicolumn{7}{|l|}{ Previously reported case } & none \\
\hline 4 & Female & 15 & poorly controlled & used & none \\
\hline
\end{tabular}

Table 1: Demographic data and condition of atopic dermatitis

\section{Clinical findings}

Of septic arthritis associated with $\mathrm{AD}$, all the patients presented with localized pain and tenderness corresponding to their sites of infection; one in the sacroiliac region (case 1), two in the knee with swelling (cases 2 and 3), and one in the hip (case 4). Two patients presented with fever $\left(>38^{\circ} \mathrm{C}\right)$; one with the knee (case 3) and one with the hip (case 4). Blood examinations showed elevated white blood cell count (WBC) and C-reactive protein (CRP) in each infection of the sacroiliac joint (case 1), the knees (cases 2 and 3), and the hip (case 4). Erythrocyte sedimentation rate (ESR) was also elevated in infections of the sacroiliac (case 1) and the hip (case 4) joints. Limited use of plain radiographs was found in the current series of joint infections. Computed tomography may be helpful to demonstrate joint effusion in the hip infection (case 4), while it failed to provide specific findings in the sacroiliac joint infection (case 1). Magnetic resonance imaging produced high sensitive and specific findings for diagnosis of infection in the sacroiliac joint (case 1) and the hip (case 4) at the early stage.

\section{Microbiology}

Identification of the pathogenic organism(s) by culture was attempted in each case. Local synovial fluid culture from the knees (cases 2 and 3) and the hip (case 4) yielded the pathogens, although no aspiration from the sacroiliac joint failed to perform microbiological investigation because of technical difficulty (case 1). No blood culture was performed in each case.

The predominant causative organism was Staphylococcus aureus (cases 2-4). Group A streptococcus probably caused the sacroiliac joint infection, following the precedent cellulitis by the bacteria (case 1). The pathogens cultured from the skin as well as the synovial fluid in the individual patients showed a similar spectrum of antibiotic sensitivities.

\section{Surgical management}

While arthroscopic irrigation was conducted in the knee infections (cases 2 and 3), open joint irrigation was performed in the hip infection (case 4).

\section{Antibiotic therapy}

Antibiotics were initially given intravenously, followed by oral antibiotics for infections in the sacroiliac joint (case 1) and the knee joints (cases 2 and 3). Intravenous antibiotics were administered for the hip infection (case 4). The spectrum of antibiotic sensitivities against the pathogens confirmed the efficacy of initial antibiotics administered in cases 2-4. In case 1, the antibiotics were selected based on the antimicrobial susceptibility to group A streptococcus from the skin lesion (Table 2). 


\begin{tabular}{|c|c|c|c|c|c|c|c|}
\hline \multirow{3}{*}{ Case } & \multicolumn{6}{|c|}{ Antibiotic therapy } & \multirow[t]{3}{*}{ Outcome } \\
\hline & \multicolumn{3}{|c|}{ intravenous } & \multicolumn{3}{|c|}{ oral } & \\
\hline & antibiotics & dose (g/day) & period (weeks) & antibiotics & dose (g/day) & period (weeks) & \\
\hline \multicolumn{8}{|c|}{ Our case series } \\
\hline 1 & flomoxef & 3 & 2 & cefdinir & 0.3 & 4 & good \\
\hline 2 & cefotiam & 3 & 2 & cefaclor & 0.75 & 3 & good \\
\hline 3 & cefazolin & 3 & 4 & levofloxacin & 0.5 & 4 & good \\
\hline \multicolumn{8}{|c|}{ Previously reported case } \\
\hline 4 & cefazolin & 2 & not described & none & & & good \\
\hline
\end{tabular}

Table 2: Antibiotic therapy and outcome

\section{Outcome}

An optimal response to treatment was obtained in septic arthritis in each patient with $\mathrm{AD}$ (cases 1-4), and no recurrence was seen at the final follow up.

\section{Discussion}

This case series is the first to emphasize the association between septic arthritis and AD. Although an entry site for articular infection is not always evident, atopic skin lesion can be a potential portal of causative bacteria. Presentation with elevated WBC, ESR, and CRP with localized pain, therefore, requires a high index of clinical suspicion for the correct diagnosis of septic arthritis in the patients with $\mathrm{AD}$. Whereas half of the children with acute hematogenous osteoarticular infections are under the age of 5, the age at onset in the current case series was between the age of 13-27 [11]. Infective endocarditis in patients with $\mathrm{AD}$ (mean age 28.4) is significantly younger than that without $\mathrm{AD}$ (mean age 53.7) [12]. Thus, the age at onset may be peculiar to hematogenous infections associated with $\mathrm{AD}$.

Intravenous antibiotics should empirically be started immediately after the clinical diagnosis of acute joint infection. A delay in antimicrobial therapy until the pathogen is identified may cause significant complications. Staphylococcus aureus was the isolated pathogen in septic arthritis in cases 2, 3 and 4, whereas group A streptococcus could be the causative organism in case 1. A high prevalence of co-infection by staphylococci and streptococci has been reported in infected AD skin lesions $[13,14]$. Taken together, the initial antibiotics that include potent cover against Staphylococcus aureus and group A streptococcus are the drug of choice for empirical therapy for septic arthritis in $\mathrm{AD}$ patients. Once the organism is isolated, antibiotics require adjustment and optimization. For confirmation of the causative bacteria, skin culture is mandatory in $\mathrm{AD}$ patients. In case of negative identification of the pathogen from culture of synovial fluid or blood, skin culture can isolate the causative organism and provide a spectrum of antibiotic sensitivities. There have been increasing reports on Methicillin-resistant Staphylococcus aureus (MRSA) infections in $\mathrm{AD}$ patients [15]. $\mathrm{AD}$ with intense and generalized erythemas, the predominant location of infection in the face, and fetid odor indicates MRSA infection [16]. It is mindful that MRSA could induce hematogenous joint infection through atopic skin lesion, which may need more intensive treatment strategy.

\section{References}

1. Goldenberg DL (1998) Septic arthritis. Lancet 351: 197-202.

2. Margaretten ME, Kohlwes J, Moore D, Bent S (2007) Does this adult patient have septic arthritis? JAMA 297: 1478-1488.

3. Till SH, Snaith ML (1999) Assessment, investigation, and management of acute monoarthritis. J Accid Emerg Med 16: 355-361.

4. Kaandorp CJ, Van Schaardenburg D, Krijnen P, Habbema JD, van de Laar MA (1995) Risk factors for septic arthritis in patients with joint disease. A prospective study. Arthritis Rheum 38: 1819-1825.

5. Kaandorp CJ, Dinant HJ, van de Laar MA, Moens HJ, Prins AP, et al. (1997) Incidence and sources of native and prosthetic joint infection: a community based prospective survey. Ann Rheum Dis 56: 470-475.

6. Gupta MN, Sturrock RD, Field M (2001) A prospective 2-year study of 75 patients with adult-onset septic arthritis. Rheumatology (Oxford) 40: 24-30.

7. Cooper C, Cawley MI (1986) Bacterial arthritis in an English health district: a 10 year review. Ann Rheum Dis 45: 458-463.

8. Söderquist B, Jones I, Fredlund H, Vikerfors T (1998) Bacterial or crystalassociated arthritis? Discriminating ability of serum inflammatory markers. Scand J Infect Dis 30: 591-596.

9. Yasuda T, Nisimatsu H (2012) Acute sacroiliac joint infection in a rugby player with atopic dermatitis. Clin J Sport Med 22: 508-510.

10. Kitamura S, Nakayama Y, Shirai Y, Hashiguchi H, Kim R (2000) Septic arthritis of the hip associated with atopic dermatitis. A case report. J Nippon Med Sch 67: 464-467.

11. Dohin B, Gillet Y, Kohler R, Lina G, Vandenesch F, et al. (2007) Pediatric bone and joint infections caused by Panton-Valentine leukocidinpositive Staphylococcus aureus. Pediatr Infect Dis J 26: 1042-1048.

12. Fukunaga N, Okada Y, Konishi Y, Murashita T, Koyama T (2013) Pay attention to valvular disease in the presence of atopic dermatitis. Circ J 77: $1862-1866$

13. Adachi J, Endo K, Fukuzumi T, Tanigawa N, Aoki T (1998) Increasing incidence of streptococcal impetigo in atopic dermatitis. J Dermatol Sci 17: 45-53.

14. Hayakawa K, Hirahara K, Fukuda T, Okazaki M, Shiohara T (2009) Risk factors for severe impetiginized atopic dermatitis in Japan and assessment of its microbiological features. Clin Exp Dermatol 34: e63-65.

15. Hon KL, Leung AK, Kong AY, Leung TF, Ip M (2008) Atopic dermatitis complicated by methicillin-resistant Staphylococcus aureus infection. J Natl Med Assoc 100: 797-800.

16. Hoeger PH (2004) Antimicrobial susceptibility of skin-colonizing S. aureus strains in children with atopic dermatitis. Pediatr Allergy Immunol 15: 474-477. 